was very sick in the morning, and vomited a good deal. She expected to be confined in two months. There was no pain in her eyes. Vision: right eye, $20 \%$; left, $20 \%$. The ophthalmoscope showed numerous exudations and hæmorrhages over both retinæ. There was a large quantity of albumen in the urine. I have heard since that the confinement was normal, and that her sight improved.

CASE VI.-M. H., aged 43, was first seen November 25th, 1881. She had had four children, one living. Last Angust, she felt the usual symptoms of pregnancy, and, a short time afterwards, a dimness of vision. Vision : right eye, $16 \frac{6}{3}$; left, $16 \frac{6}{18}$ The ophthalmoscope showed optic neuritis, with well-marked exudations and hæmorrhages. There was abundant albumen in the urine. The last report I heard was, that she was better.

CASE VII.-E. J., aged 29, was first seen May 2nd, 1881. She had been married seven years; her first child was born six years ago, still-born; the next and only child, born two months ago, lived only two days. She had had no miscarriages. Vision failed a week before confinement. When seen, it was, in the right eye, $10^{\frac{8}{8}}$ in the left, $10_{3^{6}}^{\mathrm{g}}$. No glass improved for distance. The ophthalmoscope showed numerous glistening patches and hæmorrhages around the macula in both eyes. The urine was slightly albuminous, of specific gravity 1025. Lithates were abundant. The heart was normal.

June 9th, 1881. Vision, with the right and left eyes, normal; the patches were less.

REMARK8.-Several of these cases occurred in the practice of my colleague, Dr. Little, to whose courtesy I am indebted for permission to publish them. I have also to thank our house-surgeon, Mr. Griffith, for notes of Case vir.

The prognosis in these cases is generally good, so far as the vision is concerned. Sometimes, patches of choroidal atrophy remain, and diminish the acuteness of vision considerably. As for treatment, I have generally advised the patients to be extra careful of their general health, and to take iron in some form.

I hope that these notes will induce practitioners to take careful notes of the presence of albumen during pregnancy, and the effect on the labour itself, and on the general health of the patient afterwards. The subject is one full of interest, and which I hope to discuss fuller at an early date.

\section{RHEUMATIC ENDOCARDITIS.}

\section{Bx T. J. MACLAGAN, M.D.}

To one or two points in connection with the above subject, touched on in Dr. Sansom's Lettsomian Lectures, published in recent numbers of the JOURNAL, I would like to direct attention.

In speaking of rheumatic endocarditis, Dr. Sansom quotes and endorses the two practical reasons which I give for the failure of the salicyl compounds to counteract the cardiac, as they do the arthritic, inflammation: first, that the cardiac mischief has generally commenced before the patient comes under observation; and, second, that rest, which is essential to the recovery of an inflamed organ, and which is easily got in a joint, is unattainable in the heart. The first reason makes prevention impossible; the second is a bar to successful treatment. But, though this is true, it does not express the whole truth.

That a joint generally recovers from rheumatic inflammation, and that the heart does not, is a statement which expresses the broad results of clinical experience, but expresses them in a manner which, from a pathological point of view, is bald and misleading. The truth is (and striking as the statement may appear, it is absolutely correct) that everything which recovers in a joint recovers, also, in the heart. The one structure in the heart which does not recover, the endocardium, is also the one which has no analogue in any of the structures of a joint. In studying the pathology and treatment of rheumatic endocarditis, it is essential that this point should be kept before us; if we fail to do so, we are sure to fall into error.

The parts which suffer in a joint in acute rheumatism are the fibrous ligaments and tendons, and the synovial membrane. The parts which suffer in the heart are the fibrous rings and valves, the endo-and peri-cardial membranes, and occasionally the muscular substance.

The fibrous rings and valves are similar in nature and function to the fibrous structures of a joint. Each is apt to be the seat of rheumatic inflammation and, in both, this inflammation is generally recovered from.
The pericardium finds its analogue in the synovial membrane. Fach is very vascular; each secretes a lubricating fluid; and each has for its function the facilitating the movements of a solid body. Each, too, is apt to be the seat of rheumatic inflammation; in each the inflammatory process tends to spread; and in both the tendeney is to recovery.

The endocardium has no analogue in a joint. There is nothing in a joint which bears the least resemblance to it, anatomical or physiological. It is a non-vascular membrane, in which inflammation cannot, and, as a matter of fact, does not, spread. In nature and function, it is identical with the lining membrane of the blood-vessels, with which it is structurally continuous.

When it is affected in acute rheumatism, there is no general inflammation of its surface, such as is found in the pericardium and synovial membrane; the mischief is limited to a small portion of one surface of the affected valve. I have elsewhere* shown that the occurrence of the lymphy deposit which constitutes the endocardial lesion in acute rheumatism, and its limitation to one particular portion and to one surface of the valve, are to be explained, not by the direct action of the rheumatic poison on the endocardial lining, but by the mechanical rubbing against each other of valvular segments, whose deeper fibrous structures are the seat of rheumatic inflammation and thickening. This thickening of their fibrous structure it is which makes the valves rub; and the rubbing it is which irritates and roughens the membrane that covers them externally. The inflammation and thickening of the fibrons structures of the valve may be recovered from. What is not recovered from is the roughening of, and lymphy deposit on, its non-vascular endocardial covering. This is not recovered from, because the continued action of the valve keeps up the rubbing and mechanical irritation, and because the want of blood-vessels in the endocardium prevents absorption. This is a condition over which no drug could possibly exercise any control. It is directly of mechanical, and only indirectly of rheumatic, origin.

But it is by no means certain that we may not in some cases, by the early and free administration of the salicyl compounds, prevent the inflammation, and consequent thickening, of the fibrous texture of the valve, which is the origin of all the mischief. If we see a case early enough, and give these compounds freely enough, we may prevent the heart from suffering, as we undoubtedly do prevent joints from suffering. In no given case, indeed, can we be sure of having got this result; for the absence of heart-mischief can never be demonstrated to be due to the treatment. The possibility of such a result, bowever, is worth striving for; especially as the means of attaining it are also those called for in the interests of the joints. What is wanted, is the speedy arrest of the rheumatic process. This object can be attained only by giving one of the salicyl compounds in large and frequently repeated doses.

This leads me to remark that my recommendations in this respect have not been acted up to. What I recommend is, that from 20 to 40 grains should be given every hour for six hours, or until pain is relieved (which it generally is within that time), and that the same dose should then be given every two hours till the pain is gone and the temperature at or near the normal (which is generally the case within 24 hours). After that, the same dose is given at widening intervals of three, four, and six hours, for ten or twelve days.

But instead of giving it thus freely and largely, most observers are content to give only from 15 to 20 grains every three or four hours. That is quite an inadequate dose, and not nearly enough to give the full curative effects of the drug. I would again urge the use of the dose which I recommend. I do not exceed my right in asking that my treatment should be carried out in all its details before its results are subjected to criticism.

Salicin is the preparation to which I give preference, not becanse I regard it as superior to salicylate of soda as an antirheumatic, but because it may be given in large and frequent doses without causing such disturbance of the system as not unfrequently follows the nse of the salicylate, and necessitates its suspension. My experience, too, is that those treated by salicin (which is a bitter tonic) convalesce more rapidly than those treated by the salicylate.

There is an impression abroad that it is very expensive. It is not so. Two of the chief English manufacturers of it have told me that they are prepared to supply it to hospitals and dispensaries at $10 \mathrm{~s}$. $6 \mathrm{~d}$. a pound. Convalescence is so much more rapid under its use, that I am not sure that it would not, in the long run, prove cheaper than salicylate of soda.

* On Rheumatism: its Nature, its Pathology, and its Successfu Treatment. By T. J. Maclagan, M.D. Pickering and Co. 1881. 
But, whichever is employed, let it.be given in large and frequent doses. I make this appeal in the interest of the heart as well as of the joints. Let every case of acute rheumatism be regarded and treated as one in which heart-complications may possibly be prevented, and it is probable that in some cases they will be prevented. But every hour is of importance, for it needs no argument to show that the danger to the heart is less in a case in which the course of the disease is arrested within 24 hours, than it is in one in which three or four days are expended in the process.

The fact has never been accepted by the profession that the coirse of acute rheumatism may in many cases be arrested within 24 hours of the time that treatment commences. The recognition of that fact is the keystone to all possible success in the prevention of cardiac complications.

\section{CHEMICAL ·ANALYSIS OF WATER AND WATER SUPPLY.}

BY CHARLES T. KINGZETT, F.I.C., F.C.S.

ATTexion has been recently directed in this Journal to certain investigations, from the results of which it is apparent. that "polluting material, potent for harm," may be present in a water, the analysis of which would indicate it as of "extraordinary organic purity." I believe that this fact is recognised by all chemists, and most chemists will admit that an infected water may as readily pass one method of water-analysis as another. There can be no doubt that, while chemical analysis may throw much light upon the history of any particular water, and particularly if an investigation be made (as it always should be) of the source of the water, the methods at present employed by chemists are powerless to discover the presence of disease-germs in water.

Since discussion of this subject has in a manner becn invited in thesc columns, I venture to make reference to some remarks upon Organic Matter in Water, which I published at the time when Dr. Tidy was endeavouring to obtain the confidence of chemists and others in that particular process of water-analysis which he prefers to employ. I pointed out (Chemical Nenis, vol. xli, p. 254), upon the basis of new experimental evidence, that it was possible to add a certain amount of organic matter to water, after which it would pass Dr. Tidy's process as of "great organic purity," and yet could subsequently become putrid (and therefore pernicious); in which state, judging by the sense of smell alone, or that and the use of the microscope, it would be unhesitatingly condemned by all analysts. Some time before this, I had been investigating some points in the chemical history of putrefaction (Journal of the Chomical Society, 1880, p. 15), and, in course thereof, I had come into contact with facts which seemed to me to destroy the very ground upon which the "oxygen process," as defined by Dr. Tidy rests. For instance, he says (Journal of the Chomical Society, "At any rate, it (the oxygen process) undoubtedly furnishes us with exact information as to the relative quantities of putrescent and easily oxidisable matters, and of non-putrescent or less casily oxidisable matters, present in the water." Now, my experiments clearly proved that the oxygen process can do nothing of the kind, for they, demonstrated the fact that a water may contain at one time organic matter (extract of meat) in a non-putrescent condition; and that, when these same matters-exccllent food originally-shall have become pernicious, the water will absorb far less oxygen than originally. My contention was supported by $\mathrm{Mr}$. Charles Ekin, but neither then nor since has Dr. Tidy in any way confronted this difficulty; he has contented himself with what, for all that can be proved to the contrary, seems to be a merely accidental coincidence between the general indications of the permanganate method and Dr. Frankland's combustion process as applied to water-analysis.

My experiments showed that it is possible to introduce 50 fluid grains of a putrid extract (such as $I$ had described) into a gallon of chemically pure water, without taking it out of Dr. Tidy's class of "waters of great organic purity." Similarly, 170 fluid grains could be introduced with the result of obtaining a water of "medium purity," and 255 fluid grains would only make the water of " doubtful purity." The putrid extract here referred to was swarming with organisms, and doubtless contained sepsin, which Dr. Burdon Sanderson has proved to be a blood-poison. In the face of these facts, what reliance can be placed upon the oxygen process of wateranalysis? Having asked the question, I will also answer it by saying, None.

Apart from that general information which the chemist may ob- tain from the examination of waters by the well known methods, he can get no information of a precise character. Which way then shall we take to arrive at the information we seek? At present the way is not clear, but Dr. Angus Sinith, in his extremely interesting paper (Sunitary Record, February 15th, 1883), has, I think, wandered near to it. Unfortunately, the method which he describes, admits, so far, of no quantitative expression. If we could take a measured quantity of suspected water, and introduce it into a medium in which such organisms as it contained would effect cer. tain decompositions, the products of which we could determine by measure or weight, we should be well advanced in our task, although not at the end of it; for it would still remain to ascertain if any particular product of decomposition or fermentation may be taken as indicative of the work of a particular organism, and finally, we should have to determine if such organism, being thus far identified, is to be feared as the propagating cause of a particular disease.

Forced back in this way upon well ascertained general truths, the policy to be pursued as concerns water-supply, above all things should not be experimental. We well understand the danger of contaminating our rivers with filth which breeds disease, and we also well understand the enormous difficulty and cost of finding and using another source of supply for the metropolis. In this quandary it is not surprising if we involuntarily turn to the system of water carriage now almost universally in use (thanks to engineers), as the source of all this trouble, and I, for one, think that the shortest way out of the difficulty is to return to the dry closet system, taking advantage of chemical means (of which there are plenty) to prevent the excreta from becoming a source of pollution and disease. Our water-courses would then, in due time, reassume their original purity ; we should be no longer exposed to the puffs of gas, loaded with disease-germs and poisons, which escape from the sewers into our houscs, and from the ventilators into our streets, and we should cease to commit the enormous folly of throwing into the sea those valuable substances which, in the natural order of things, should be immediately returned to the soil which grows our food.

\section{SURGICAL MEMORANDA.}

\section{GUNSHOT-WOUND $\overline{O H^{\top}}$ THE ABDOMEN.}

IN his very interesting history of a case of penetrating gunshotwound of the abdomen, published in the JoURNAL of Miny 31 st, Professor Buchanan states that he has been unable to find an example of " $a$ bullet entering near the umbilicus, and making the half circuit of the body." As I came across a case somewhat of that nature a few years ago, I venture to send the following brief particulars.

During the engagement which followed the successful surprise of the village of Suppri, on the north-west frontier of India, on February 15th, 1878; a sowar of the Queen's Own Corps of Guides was struck by a ballet in the back. I saw the man, who was but two or three yards from me at the time, stagger, and expected to see him fall. He remained standing, however, and called out to me that he was wounded. Having given'directions to the hospital-assistant as to his disposal, and mentally noting that the wound could be only a slight one on account of his being able to walk down a steep hillside with but little help, my attention was soon directed elsewhere. Some hours later, when the force had recrossed the frontier, and I could make a detailed examination of the wounded, I found the sowar above mentioned in bed, lying on his back, with his legs drawn up, and his face cxpressive of acute suffering. My first impression on seeing him in this state was, that the bullet had penetrated the abdominal cavity. There was not, however, the marked collapse usually attending sq grave an injury. The bullet had penetrated the lumbar muscles on the right side of the spine, an inch from the middle line. Having stripped the abdomen with a view to a careful examination, as my fingers passed over the hepatic region, a slight prominence was encountered, of such firm consistence as to at once lead to the conclusion that it was the bullet. A small incision over the spot was at once made, and a round bullet extracted, to the great delight of the patient, and the immediate relief of his suffering. Both wounds healed in a few days, without any suppuration of the track made by the bollet. In this case, a round bullet, travelling most probably with slight velocity, was deflected by the lumbar fascia, and very nearly made the half circuit of the body, as the site of extraction was two inches from the middle line in front, immediately below the false ribs.

A. S. Mallins, A.B., M.B., M.Ch., late Surgeon A.M.D., Walton, Norfolk. 